\title{
BEST SIMULTANEOUS DIOPHANTINE APPROXIMATIONS. I. GROWTH RATES OF BEST APPROXIMATION DENOMINATORS
}

BY

\author{
J. C. LAGARIAS
}

\begin{abstract}
ABSTRACr. This paper defines the notion of a best simultaneous Diophantine approximation to a vector $\alpha$ in $R^{n}$ with respect to a norm $\|\cdot\|$ on $R^{n}$. Suppose $\alpha$ is not rational and order the best approximations to $\alpha$ with respect to $\|\cdot\|$ by increasing denominators $1=q_{1}<q_{2}<\cdots$. It is shown that these denominators grow at least at the rate of a geometric series, in the sense that
\end{abstract}

$$
g(\alpha,\|\cdot\|)=\liminf _{k \rightarrow \infty}\left(q_{k}\right)^{1 / k} \geqslant 1+\frac{1}{2^{n+1}} .
$$

Let $g(\|\cdot\|)$ denote the infimum of $g(\alpha,\|\cdot\|)$ over all $\alpha$ in $R^{n}$ with an irrational coordinate. For the sup norm $\|\cdot\|_{s}$ on $R^{2}$ it is shown that $g\left(\|\cdot\|_{s}\right) \geqslant \theta=1.270^{+}$ where $\theta^{4}=\theta^{2}+1$.

1. Introduction. The problem of simultaneous Diophantine approximation is that of approximating a vector of two or more real numbers by a vector of rational numbers having the same denominator. Two of the most fundamental questions in this subject concern how well such vectors can be approximated, and how often good approximations occur. It is well known that the analogous questions for the approximation of a single real number $\theta$ can be answered in terms of the continued fraction expansion of $\theta$. Concerning simultaneous Diophantine approximation Davenport [7] remarks: "In any deeper investigation of the problem of simultaneous Diophantine approximation we are greatly handicapped by the absence of a full analogue of the continued fraction process. There are several analogues, but they all suffer from one of two defects: either they give much poorer approximations than we known to exist, or they involve a series of operations that can be carried out on given $\theta_{1}, \ldots, \theta_{n}$ but cannot be used to define $\theta_{1}, \ldots, \theta_{n}$, because we do not know the limitations to which any such sequence is subject." As a consequence much less is known than in the one-dimensional case. For example, it remains a notoriously difficult unsolved problem to determine the two-dimensional Diophantine approximation constant $(\operatorname{see}[1,3,4,6])$.

This paper considers the notion of a best simultaneous approximation to a vector $\alpha$ in $R^{n}$ with respect to a norm $\|\cdot\|$ on $R^{n}$. To define this notion precisely, let $\alpha=\left(\alpha_{1}, \ldots, \alpha_{n}\right)$ and let $\|\cdot\|$ denote a norm on $R^{n}$. Given an integer denominator

Received by the editors July 15, 1980 and, in revised form, March 3, 1981.

1980 Mathematics Subject Classification. Primary 10F10.

Key words and phrases. Simultaneous Diophantine approximation.

(C)1982 American Mathematical Society 0002-9947/82/0000-0008/\$03.00 
$q>0$ we measure the closeness of approximation possible by rationals of denominator $q$ by

$$
\delta_{q}=\delta_{q}(\alpha)=\underset{\left(p_{1}, \ldots, p_{n}\right) \in Z^{n}}{\operatorname{MIN}}\left\|\left(q \alpha_{1}-p_{1}, \ldots, q \alpha_{m}-p_{m}\right)\right\| .
$$

The best simultaneous approximation denominators (BSAD's) $q_{k}=q_{k}(\alpha)$ to the vector $\alpha$ (with respect to the norm $\|\cdot\|$ ) are inductively defined by $q_{1}=1$ and by taking $q_{k}$ to be the least positive integer such that $\delta_{q_{k}}<\delta_{q_{k-1}}$. The corresponding best simultaneous approximations (BSA's) $\mathbf{v}_{k}$ are the integer vectors

$$
\mathbf{v}_{k}=\mathbf{v}_{k}(\alpha)=\left(q_{k}, p_{k, 1}, \ldots, p_{k, n}\right)
$$

in $Z^{n+1}$ where $\left(p_{k, 1}, \ldots, p_{k, n}\right)$ achieves the minimum in (1.1) for $q_{n}$. (In case more than one vector $\left(p_{1}, \ldots, p_{n}\right)$ achieves the minimum in (1.1) for a given $q_{k}$, we simply choose one in an arbitrary manner to obtain (1.2). This situation can occur for only finitely many BSAD's; see the discussion at the beginning of $\$ 2$.) In general we call any vector $\mathbf{v}=\left(q, p_{1}, \ldots, p_{n}\right)$ minimizing (1.1) an approximation vector, and

$$
\mathbf{R}(\mathbf{v})=\left(q \alpha_{1}-p_{1}, \ldots, q \alpha_{n}-p_{n}\right)
$$

its corresponding vector of approximation remainders. Most previous work on simultaneous approximation measures closeness of approximation using the sup norm $\|\cdot\|$, given by

$$
\left\|\left(x_{1}, \ldots, x_{n}\right)\right\|_{s}=\underset{1 \leqslant i \leqslant n}{\operatorname{MAX}}\left|x_{j}\right| .
$$

The Euclidean norm $\|\cdot\|_{e}$, given by

$$
\left\|\left(x_{1}, \ldots, x_{n}\right)\right\|_{e}=\left(x_{1}^{2}+\cdots+x_{n}^{2}\right)^{1 / 2},
$$

has also been considered, cf. $[2,8,13]$.

Various notions of "best approximation" have previously appeared in the literature. Davenport and Schmidt $[9,10]$ introduced and successfully made use of an analogous notion of a best approximation to a single linear form. More recently Cusick [5] and Dubois [11] considered the problem of finding such approximations for a ternary linear form. Brentjes [2], Dubois and Rhin [12], and Jurkat, Kratz and Peyerimhoff [13] have recently considered best simultaneous approximations in $R^{2}$ with respect to the Euclidean norm.

This paper is the first of a series of papers studying properties of best simultaneous approximations. In this paper we consider the problem of how often best approximations occur, as measured by the rate of growth of the best approximation denominators.

The rate of growth of best approximation denominators is well understood in the one-dimensional case. Given an irrational number $\theta$, let $\mathbf{v}_{k}=\left(q_{k}, p_{k}\right)$ denote the $k$ th best approximation vector in the sup norm (the only norm that exists on $R^{1}$ ). It is well known (Lang [17, p. 10]) that $p_{k} / q_{k}$ is just the $k$ th convergent of the ordinary continued fraction expansion of $\theta$. In particular

$$
q_{k} \geqslant q_{k-1}+q_{k-2}
$$


and as a consequence

$$
g(\theta)=\liminf _{k \rightarrow \infty}\left(q_{k}\right)^{1 / k} \geqslant \frac{1+\sqrt{5}}{2} .
$$

This inequality asserts that the one-dimensional best approximation denominators grow at least at the rate of a geometric progression. The value $g(\theta)=(1+\sqrt{5}) / 2$ is attained for $\theta=(1+\sqrt{5}) / 2$ (and in fact for uncountably many other real numbers).

It is easy to prove that a geometric growth rate occurs in all dimensions.

THEOREM 1.1. There is a constant $g_{n}>1$ depending only on $n$, such that for any norm $\|\cdot\|$ on $R^{n}$ and any $\alpha \in R^{n}$ with $\alpha \notin Q^{n}$,

$$
g(\alpha,\|\cdot\|)=\liminf _{k \rightarrow \infty} q_{k}(\alpha)^{1 / k} \geqslant g_{n}>1 .
$$

We can take $g_{n}=1+1 / 2^{n+1}$.

This theorem allows us to define the growth rate constant $G(\|\cdot\|)$ for a norm $\|\cdot\|$ by

$$
G(\|\cdot\|)=\inf _{\alpha \in R^{n}} g(\alpha,\|\cdot\|)
$$

and the $n$-dimensional growth rate constant

$$
G(n)=\inf _{\|\cdot\| \text { on } R^{n}} G(\|\cdot\|) .
$$

The main result of this paper is an improvement of the lower bound of Theorem 1.1 for the growth rate constant of the sup norm $\|\cdot\|_{s}$ on $R^{2}$. We show that

$$
G\left(\|\cdot\|_{s}\right) \geqslant \theta=1.2720^{+} \text {, }
$$

where $\theta$ is the largest real root of $\theta^{4}=\theta^{2}+1$. In the course of establishing (1.11) we prove an "isolation" result which restricts the values of consecutive best approximation denominators which are close together.

THEOREM 1.2. Let $\alpha \in R^{2}$ have both coordinates irrational. Let $q_{k}, q_{k+1}, q_{k+2}, q_{k+3}$ be four consecutive best approximation denominators to $\alpha$ with respect to the sup norm $\|\cdot\|_{s}$, and suppose that

$$
q_{k+3}<q_{k+1}+q_{k}
$$

Then

$$
q_{k+3}=q_{k+2}+q_{k+1}-q_{k} \text {. }
$$

There exist $\alpha \in R^{2}$ for which (1.12) occurs. (See Lagarias [14, Table II] for an analogous example of (1.12) in the case of Euclidean norm best approximations.) Theorem 1.2 may be compared to the one-dimensional situation, where for three consecutive best approximation denominators one has $q_{k+2}=a q_{k+1}+q_{k}$ for some positive integer $a$.

Brentjes [2, Example 4] and Lagarias [16] show that

$$
G\left(\|\cdot\|_{e}\right) \leqslant \eta=1.3247^{+}
$$

where $\|\cdot\|_{e}$ is the Euclidean norm and $\eta$ is the real root of $\eta^{3}=\eta+1$. This fact, 
together with the form of Theorem 1.2 and a variety of other evidence, suggests the following conjecture.

Conjecture. $G(2)=\eta=1.3247^{+}$where $\eta$ is the real root of $\eta^{3}=\eta+1$.

Some results of this paper were announced in [14]. Other results on best simultaneous Diophantine approximations appear in [14]-[16].

2. Growth rates for best simultaneous approximations. We first make some preliminary remarks about norms and the uniqueness of best approximation vectors. A function $\|\cdot\|: R^{n} \rightarrow R^{n}$ is a norm provided

(i) $\|\mathbf{x}\| \geqslant 0$, and $\|\mathbf{x}\|=0 \Rightarrow \mathbf{x}=\mathbf{0}$.

(ii) $\left\|\mathbf{x}_{1}+\mathbf{x}_{2}\right\| \leqslant\left\|\mathbf{x}_{1}\right\|+\left\|\mathbf{x}_{2}\right\|$.

(iii) $\|\lambda \mathbf{x}\|=|\lambda|\|\mathbf{x}\|$ for $\lambda \in R$.

The unit ball $B=\{\mathbf{x} \mid\|\mathbf{x}\|<1\}$ of a norm is a centrally symmetric convex body. Conversely, any such body $B$ determines a unique norm on $R^{n}$ for which it is the unit ball. The norms $\|\cdot\|$ and $\lambda\|\cdot\|$ for any $\lambda>0$ determine the same set of BSAD's and BSA's. Therefore we may when necessary deal without loss of generality with scaled norms having the properties

(2.1) (i) $\mathbf{x} \in Z^{n}$ and $\mathbf{x} \neq \mathbf{0} \Rightarrow\|\mathbf{x}\| \geqslant 1$,

(ii) there is an $\mathbf{x} \in Z^{n}$ with $\|\mathbf{x}\|=1$.

Both the Euclidean and sup norms are scaled norms.

LEMMA 2.1. (i) For a scaled norm, a vector $\alpha$, and any denominator $q$ with $\delta_{q}(\alpha)<\frac{1}{2}$ there is a unique choice of approximation vector $\mathbf{v}=\left(q, p_{1}, \ldots, p_{n}\right)$ with denominator $q$.

(ii) For any norm $\|\cdot\|$ and any $\varepsilon>0$ there is a bound $K=K(\|\cdot\|)$ such that $\delta_{q}(\alpha)<\varepsilon$ for all $\alpha$ and all best simultaneous approximation denominators $q>K$.

Proof. (i) Suppose $\mathbf{v}$ is one such approximation vector, so that $\|\mathbf{R}(\mathbf{v})\|=\delta_{q}<\frac{1}{2}$. For any other $\mathbf{v}^{*},\left\|\mathbf{R}\left(\mathbf{v}^{*}\right)\right\|=\mathbf{R}(\mathbf{v})+\mathbf{x}$ for some nonzero $\mathbf{x} \in \mathbf{Z}^{n}$. Then by the triangle inequality

$$
\left\|\mathbf{R}\left(\mathbf{v}^{*}\right)\right\|=\|\mathbf{x}+\mathbf{R}(\mathbf{v})\| \geqslant\|\mathbf{x}\|-\|\mathbf{R}(\mathbf{v})\|>\frac{1}{2}
$$

since $\|\mathbf{x}\|>1$. Hence $\left\|R\left(\mathbf{v}^{*}\right)\right\| \neq \delta_{q}$, and $\mathbf{v}$ is unique.

(ii) It is well known that any norm $\|\cdot\|$ on $R^{n}$ is compatible with the sup norm $\|\cdot\|_{s}$ in the sense that there are positive constants $c_{1}, c_{2}$ (depending on $\|\cdot\|$ ) such that

$$
c_{1}\|\mathbf{x}\| \leqslant\|\mathbf{x}\|_{s} \leqslant c_{2}\|\mathbf{x}\|
$$

for all $\mathbf{x} \in R^{n}$. Dirichlet's theorem on simultaneous approximation asserts that for the sup norm $\|\cdot\|_{s}$ and any $\alpha \in R^{n}$ for any given integer $K$ there is some $q$ with $1 \leqslant q \leqslant K$ such that

$$
\delta_{q}=\delta_{q}(\alpha) \leqslant K^{1 / n}
$$

Choosing $K=\left(\varepsilon c_{1}\right)^{-n}$ in (2.4) and applying (2.3) shows there is some $q<K$ with $\delta_{q}<\varepsilon$. This implies (ii). 
In view of Lemma 2.1, whenever $\delta_{q}<\frac{1}{2}$ we may unambiguously use the notations

$$
\mathbf{v}(q)=\left(q, p_{1}, \ldots, p_{n}\right), \quad \mathbf{R}(q)=\left(q \alpha_{1}-p_{1}, \ldots, q \alpha_{n}-p_{n}\right)
$$

for the approximation vector and approximation remainder vector, respectively.

The lower bound of Theorem 1.1 is a consequence of the following result, which gives "local" inequality satisfied by the best simultaneous approximation denominators. It is analogous to (1.6). The proof is that of a referee, and sharpens the author's original result, cf. [14].

TheOREM 2.2. For any norm $\|\cdot\|$ on $R^{n}$, and any vector $\alpha \in R^{n}-Q^{n}$, the best simultaneous approximation denominators $q_{k}$ to $\alpha$ satisfy

$$
q_{k+2^{n+1}} \geqslant 2 q_{k+1}+q_{k} \text {. }
$$

Proof. We argue by contradiction. Suppose

$$
q_{k+2^{n+1}}<2 q_{k+1}+q_{k} .
$$

We examine the approximation vectors $\mathbf{v}_{k+j}(\bmod 2)$ for $0 \leqslant j \leqslant 2^{n+1}$. By the pigeonhole principle there exist $i, j$ with $i>j$ such that

$$
\mathbf{v}_{k+i} \equiv \mathbf{v}_{k+j}(\bmod 2) \text {. }
$$

Then $\mathbf{v}=\frac{1}{2}\left(\mathbf{v}_{k+i}-\mathbf{v}_{k+j}\right) \in \mathbf{Z}^{n}$ has denominator $q=\frac{1}{2}\left(q_{k+i}-q_{k+j}\right)$ and by (2.7) we have

$$
0<q \leqslant \frac{1}{2}\left(q_{k+2^{n+1}}-q_{k}\right)<q_{k+1}
$$

But

$$
\begin{aligned}
\delta_{q} & \leqslant\|\mathbf{R}(\mathbf{v})\| \leqslant \frac{1}{2}\left(\left\|\mathbf{R}\left(\mathbf{v}_{k+i}\right)\right\|+\left\|\mathbf{R}\left(\mathbf{v}_{k+j}\right)\right\|\right) \\
& \leqslant \frac{1}{2}\left(\boldsymbol{\delta}_{k+i}+\delta_{k+j}\right) \leqslant \frac{1}{2}\left(\delta_{k}+\delta_{k+1}\right)<\boldsymbol{\delta}_{k} .
\end{aligned}
$$

This contradicts the definition of $q_{k+1}$.

Proof of TheOREM 1.1. Let $\phi$ be the largest positive root of

$$
f(x)=x^{2^{n+1}}-2 x-1=0 .
$$

Then $\phi>1+1 / 2^{n+1}=\Delta$ since $\Delta^{2^{n+1}}<e<2 \Delta+1$, so $f(\Delta)<0$. Pick $K_{0}$ as in Theorem 2.3 and choose a positive $\varepsilon$ small enough that

$$
q_{K_{0}+j} \geqslant \varepsilon \phi^{j} \text { for } 0 \leqslant j \leqslant 2^{n+1}-1 \text {. }
$$

Then using (2.6) of Theorem 2.2, we conclude

$$
q_{K_{0}+j} \geqslant \varepsilon \phi^{j}
$$

holds for all $j \geqslant 0$, using (2.12) and induction on $j$. Then (2.13) implies

$$
\liminf _{k \rightarrow \infty}\left(q_{k}\right)^{1 / k} \geqslant \phi>1+\frac{1}{2^{n+1}},
$$

completing the proof. 
THEOREM 2.3. Let $\|\cdot\|_{s}$ be the sup norm on $R^{n}$. For any vector $\alpha \in R^{n}-Q^{n}$, its best simultaneous approximation denominators satisfy

$$
q_{k+2^{n}} \geqslant q_{k}+q_{k+1}
$$

for all sufficiently large $k$, depending on $\alpha$. If $\alpha$ has no rational coordinates, then (2.15) is true for all $k \geqslant 1$.

Proof. Associate to each approximation remainder vector $\mathbf{R}(q)$ a vector of signs \pm 1 via

$$
\operatorname{sgn} \mathbf{R}(q)=\left(\operatorname{sgn}\left(q \alpha_{1}-p_{1}\right), \ldots, \operatorname{sgn}\left(q \alpha_{n}-p_{n}\right)\right) .
$$

If some $q \alpha_{i}-p_{i}=0$, its sign may be chosen arbitrarily.

There are $2^{n}$ possible distinct sign vectors, so by the pigeonhole principle there must be two denominators among the $2^{n}+1$ denominators $q_{k}, q_{k+1}, \ldots, q_{k+2^{n}}$ which have identical sign vectors. Call these $q_{i}, q_{j}$ and order them so that $q_{i}>q_{j}$.

We suppose $q_{i}<q_{j+1}+q_{j}$ so that

$$
q=q_{i}-q_{j}<q_{j+1}
$$

and derive a contradiction. We use the notation $r_{i}(q)=q \alpha_{i}-p_{i}$ for the $i$ th component of the approximation remainder vector $\mathbf{R}(q)$. Since the signs of $r_{k}\left(q_{i}\right)$, $r_{k}\left(q_{j}\right)$ agree for all $k$,

$$
\left|r_{k}(q)\right|=\left|r_{k}\left(q_{i}\right)-r_{k}\left(q_{j}\right)\right| \leqslant \operatorname{MAX}\left(\left|r_{k}\left(q_{i}\right)\right|,\left|r_{k}\left(q_{j}\right)\right|\right) \leqslant \delta_{q_{j}} .
$$

Hence $\delta_{q} \leqslant \delta_{q_{j}}$. We will sharpen this to

$$
\delta_{q}<\delta_{q_{j}}
$$

Equality can occur in the set of inequalities leading to (2.17) only if $r_{k}\left(q_{i}\right)=0$ for at least one of the $k$ for which $\left|r_{k}\left(q_{j}\right)\right|$ attains its maximum. This requires $\alpha_{k} \in Q$, so (2.18) always holds when all coordinates of $\alpha$ are irrational. In the remaining case, we recall that Dirichlet's principle gives

$$
\delta_{q_{j}}<\left(q_{j}\right)^{-1 / n}
$$

Let $M$ be the greatest common denominator of all the rational coordinates $\alpha_{k}$ of $\alpha$. For a rational $\alpha_{k}$ either $r_{k}(q)=0$ or $\left|r_{k}(q)\right|>M^{-1}$. If $q_{j}>M^{n}$ then (2.17) together with (2.19) forces $r_{k}\left(q_{j}\right)=0$. Henceforth suppose $q_{j}>M^{n}$, a condition eliminating a finite number of $q_{j}$. In this case $r_{k}\left(q_{i}\right)=0$ implies $r_{k}\left(q_{j}\right)=0$, so that $\delta_{q_{j}}=0$ (since $k$ was chosen to maximize $\left|r_{k}\left(q_{j}\right)\right|$ ) and $\alpha \in Q^{n}$, a contradiction. So (2.18) holds in this case as well. But (2.16) implies $\delta_{q} \geqslant \delta_{q_{j}}$ and this contradicts (2.18). Hence $q \geqslant q_{j+1}+q_{j}$. Finally

$$
q_{k+2^{n}} \geqslant q_{i} \geqslant q_{j+1}+q_{j} \geqslant q_{k+1}+q_{k} .
$$

REMARK. This proof also shows that two consecutive best approximations with denominators $q_{k}, q_{k+1}$ must have different sign vectors $\operatorname{sgn} \mathbf{R}\left(q_{k}\right)$, sgn $\mathbf{R}\left(q_{k+1}\right)$.

The following corollary has a proof similar to that of Theorem 1.1.

COROllary 2.4. For the sup norm $\|\cdot\|_{s}$ on $R^{n}$, the minimal growth rate of best simultaneous approximation denominators satisfies $G\left(\|\cdot\|_{s}\right) \geqslant \theta$ where $\theta>1$ satisfies $\theta^{2^{n}}=\theta+1$. 
3. Growth rates: 2-dimensional sup norm case. The object of this section is to improve on Theorem 2.3 in the two-dimensional sup norm case. To accomplish this we first prove a result which implies Theorem 1.2 of the introduction.

THEOREM 3.1. Consider the best simultaneous approximation denominators $q_{k}$ to $\alpha=\left(\alpha_{1}, \alpha_{2}\right)$ with respect to the sup norm.

(i) If $\alpha$ has exactly one irrational coordinate, then for all sufficiently large $k$ depending on $\alpha$,

$$
q_{k+2} \geqslant q_{k+1}+q_{k}
$$

(ii) If $\alpha$ has both coordinates irrational, then

$$
q_{k+3}<q_{k+1}+q_{k}
$$

can only occur when

$$
q_{k+3}=q_{k+2}+q_{k+1}-q_{k} \text {. }
$$

Furthermore (3.2) can never hold for two consecutive values of $k$.

Proof. (i) Suppose $\alpha$ has exactly one rational coordinate. Without loss of generality we may suppose this is $\alpha_{1}$, and that it has denominator $M$. From the proof of Theorem 2.3, $r_{1}\left(q_{k}\right)=0$ whenever $q_{k} \geqslant M^{2}$. We suppose this is true and specify $\operatorname{sgn} r_{1}\left(q_{k}\right)=+1$. Then any three consecutive best approximations $\mathbf{v}_{k}, \mathbf{v}_{k+1}, \mathbf{v}_{k+2}$ have two with the same sign vector. The proof of Theorem 2.3 applies to give $q_{k+2} \geqslant q_{k+1}+q_{k}$, the desired result. (In general if a $d$-vector $\alpha$ has $k$ rational entries, the inequality (2.15) of the $n=d-k$ dimensional case applies.)

(ii) Assume both coordinates of $\alpha$ are irrational. Let $q_{k}, q_{k+1}, q_{k+2}, q_{k+3}$ be four consecutive best approximation denominators, and suppose that (3.2) holds. In that case the sign vectors $\operatorname{sgn} \mathbf{R}\left(q_{k+i}\right)$ for $0 \leqslant i \leqslant 3$ must assume all four possible sets of signs. If not the proof of Theorem 2.3 would yield

$$
q_{k+3} \geqslant q_{k+1}+q_{k}
$$

contradicting (3.2).

The four approximation remainders $\mathbf{R}\left(q_{k+i}\right)(i=1, \ldots, 4)$ lie in the four different quadrants. Let $\mathbf{r}_{1}, \ldots, \mathbf{r}_{4}$ be these four remainders, reordered such that $\mathbf{r}_{i}$ lies in the $i$ th quadrant. Then put $\mathbf{w}_{1}=-\mathbf{r}_{3}, \mathbf{w}_{2}=\mathbf{r}_{2}, \mathbf{w}_{3}=-\mathbf{r}_{3}, \mathbf{w}_{4}=\mathbf{r}_{4}$, so that $\mathbf{w}_{i}$ again lies in the $i$ th quadrant.

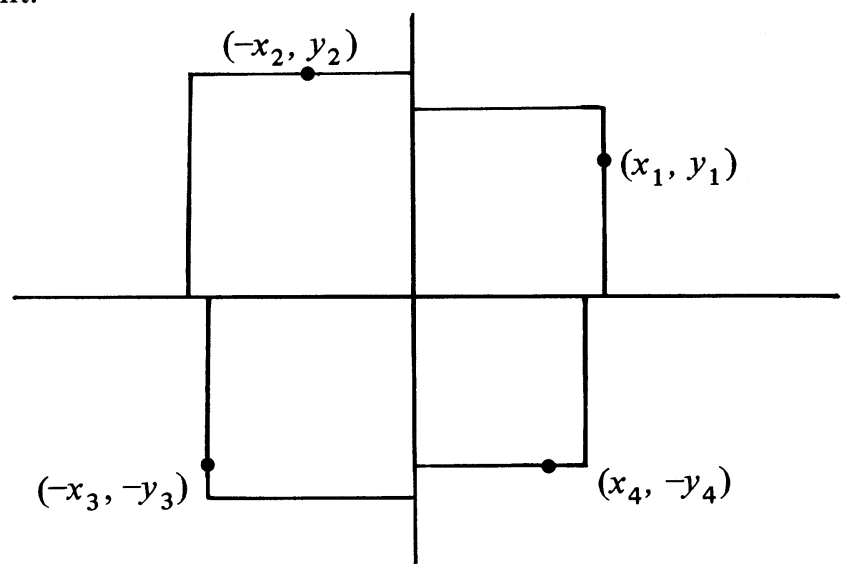

FIGURE 1. Approximation remainder vectors 
As pictured in Figure 1, we have

$$
\mathbf{w}_{1}=\left(x_{1}, y_{1}\right), \quad \mathbf{w}_{2}=\left(-x_{2}, y_{2}\right), \quad \mathbf{w}_{3}=\left(-x_{3},-y_{3}\right), \quad \mathbf{w}_{4}=\left(x_{4},-y_{4}\right)
$$

with positive $x_{i}, y_{i}$. (None can be equal to 0 because $\alpha_{1}, \alpha_{2}$ are both irrational.) These four points $\mathbf{w}_{i}$ are the approximation remainder vectors corresponding to denominators $-q_{1}, q_{2},-q_{3}, q_{4}$, respectively.

Consider the quadrants I and II. Set $q=\left|-q_{1}+q_{2}\right|$. Then

$$
0<q \leqslant q_{k+3}-q_{k}<q_{k+1} \text {. }
$$

This implies

$$
\delta_{q} \geqslant \delta_{q_{k}}
$$

But $\delta_{q}=\operatorname{MAX}\left(\left|r_{1}(q)\right|,\left|r_{2}(q)\right|\right)$, and

$$
\left|r_{1}(q)\right| \leqslant\left|x_{1}-x_{2}\right|<\operatorname{MAX}\left(\delta_{1}, \delta_{2}\right) \leqslant \delta_{q_{k}} .
$$

Then (3.7) forces

$$
y_{1}+y_{2} \geqslant\left|r_{2}(q)\right| \geqslant \delta_{q_{k}} .
$$

Since $y_{1}, y_{2}$ lie on squares of sides $\delta_{1}, \delta_{2}$ we obtain

$$
y_{1}+y_{2} \leqslant \delta_{1}+\delta_{2} \leqslant \delta_{q_{k}}+\delta_{q_{k+1}} .
$$

Putting these last two inequalities together we have

$$
\delta_{q_{k}}+\delta_{q+1} \geqslant y_{1}+y_{2} \geqslant \delta_{q_{k}} .
$$

Identical arguments for the other three sets of adjacent quadrants give

$$
\begin{aligned}
& \delta_{q_{k}}+\delta_{q_{k+1}} \geqslant x_{2}+x_{3} \geqslant \delta_{q_{k}}, \\
& \delta_{q_{k}}+\delta_{q_{k+1}} \geqslant y_{3}+y_{4} \geqslant \delta_{q_{k}}, \\
& \delta_{q_{k}}+\delta_{q_{k+1}} \geqslant x_{4}+x_{1} \geqslant \delta_{q_{k}} .
\end{aligned}
$$

We proceed to sharpen some of (3.11)-(3.14) to strict inequalties. Three of the left-hand inequalities of (3.11)-(3.14) are strict, because such an inequality can be an equality only when $\delta_{i}, \delta_{i+1}$ are $\delta_{q_{k}}, \delta_{q_{k+1}}$ and this can happen for at most one pair of adjacent quadrants. Suppose this occurs. We show the right-hand inequality of the equation corresponding to the opposite pair of quadrants must then be strict. The opposite pair of quadrants must have $\delta_{q_{k+2}}, \delta_{q_{k+3}}$, so that the corresponding $q$ in (3.6) has $q \leqslant q_{k+3}-q_{k+2}<q_{k}$. Consequently

$$
\delta_{q} \geqslant \delta_{q_{k-1}}>\delta_{q_{k}},
$$

and that sharpens the right side of the corresponding (3.10) to strict inequality.

Next set

$$
q=\left|-q_{1}+q_{2}-q_{3}+q_{4}\right|
$$

Now

$$
q \leqslant q_{k+3}+q_{k+2}-q_{k+1}-q_{k}<q_{k+2}
$$

by hypothesis (3.2). Suppose $q>0$. Then

$$
\delta_{q} \geqslant \delta_{q_{k+1}} .
$$


On the other hand

$$
\begin{aligned}
\left|r_{1}(q)\right| & \leqslant\left|x_{1}-x_{2}-x_{3}+x_{4}\right|=|| x_{1}+x_{4}|-| x_{2}+x_{3}|| \\
& <\left(\delta_{q_{k+1}}+\delta_{q_{k}}\right)-\delta_{q_{k}}=\delta_{q_{k+1}}
\end{aligned}
$$

using (3.12), (3.14). The preceding remarks about strict inequalities were used to guarantee strict inequality in the last line. Similarly

$$
\begin{aligned}
\left|r_{2}(q)\right| & \leqslant\left|y_{1}+y_{2}-y_{3}-y_{4}\right|=|| y_{1}+y_{2}|-| y_{3}+y_{4}|| \\
& <\left(\delta_{q_{k+1}}+\delta_{q_{k}}\right)-\delta_{q_{k}}=\delta_{q_{k+1}}
\end{aligned}
$$

using (3.11) and (3.13). Then (3.18) and (3.19) contradict (3.17).

Consequently we must have $q=0$ in (3.15). Now $q_{k}<q_{k+1}<q_{k+2}<q_{k+3}$ implies

$$
q_{k+3}+q_{k+2}-q_{k+1}-q_{k}>0, \quad q_{k+3}-q_{k+2}+q_{k+1}-q_{k}>0 .
$$

Hence $q=0$ can occur only if

$$
q_{k+3}-q_{k+2}-q_{k+1}+q_{k}=0,
$$

which gives (3.3).

To complete the proof of (ii), we note further that in the case $q=0$ comparing the definition (3.15) with (3.20) shows that the remainder vectors $\mathbf{R}\left(q_{k}\right)$ and $\mathbf{R}\left(q_{k+1}\right)$ must be in adjacent quadrants. It also shows the remainder vectors $\mathbf{R}\left(q_{k+1}\right)$ and $\mathbf{R}\left(q_{k+2}\right)$ must be in opposite quadrants. As a consequence, if (3.2) holds for $k$, it cannot hold for $k+1$, for then $\mathbf{R}\left(q_{k+1}\right)$ and $\mathbf{R}\left(q_{k+2}\right)$ would have to be in adjacent quadrants, a contradiction. (Here note the remainder vectors $\mathbf{R}\left(q_{k+1}\right), \mathbf{R}\left(q_{k+2}\right)$ cannot be on the quadrant boundaries because $\alpha$ has both coordinates irrational.)

The proof of the following corollary is due to A. M. Odlyzko.

COROllaRY 3.2. For the sup norm $\|\cdot\|_{s}$ on $R^{2}$ the minimal growth rate for best simultaneous approximation denominators satisfies

$$
G\left(\|\cdot\|_{s}\right) \geqslant \theta
$$

where $\theta=1.2720^{+}$is the largest real root of $\theta^{4}=\theta^{2}+1$.

Proof. If $\alpha \in R^{2}-Q^{2}$ has one irrational coordinate, then by Theorem 3.1(i) its sup norm BSAD's have growth rate at least $\phi=1.6180^{+}$, the golden ratio. If $\alpha$ has both coordinates irrational then using Theorem 3.1(ii) it is easy to establish by induction that

$$
q_{k} \geqslant u_{k}
$$

where $u_{1}=1, u_{2}=2, u_{3}=3, u_{4}=4$, and $u_{k}=u_{k-2}+u_{k-4}$. To see this, Theorem 3.1(ii) asserts for each $k$ that either

$$
q_{k} \geqslant q_{k-2}+q_{k-3}
$$

holds, or else

$$
q_{k}=q_{k-1}+q_{k-2}-q_{k-3}, \quad q_{k-1} \geqslant q_{k-3}+q_{k-4}
$$


both hold. Since the $q_{k}$ are increasing, both (3.23) and (3.24) imply

$$
q_{k} \geqslant q_{k-2}+q_{k-4}
$$

The induction follows via (3.25). Then

$$
\liminf _{k \rightarrow \infty}\left(q_{k}\right)^{1 / k} \geqslant \liminf _{k \rightarrow \infty}\left(u_{k}\right)^{1 / k}=\theta
$$

where $\theta=\sqrt{\phi}$ is the largest real root of $\theta^{4}=\theta^{2}+1$.

ACKNOWLEDGEMENTS. I am indebted to W. W. Adams for introducing me to these problems, for many helpful conversations, and for supplying references to the literature. I thank W. W. Adams, F. Beukers and M. Razar for commenting on drafts of this paper. I thank a referee for the sharpened proof of Theorem 2.2 and A. M. Odlyzko for the proof of Corollary 3.2.

\section{REFERENCES}

1. W. W. Adams, Simultaneous Diophantine approximations and cubic irrationals, Pacific J. Math. 30 (1969), 1-14.

2. A. J. Brentjes, A two-dimensional continued fraction algorithm for best approximations with an application to cubic number fields, J. Reine Angew. Math. 326 (1981), 18-44.

3. J. W. S. Cassels, Simultaneous Diophantine approximation, J. London Math. Soc. 30 (1955), $119-121$.

4. T. W. Cusick, Formulas for some Diophantine approximation constants. II, Acta Arith. 26 (1974), 117-128.

5. __ Best Diophantine approximations for ternary linear forms, J. Reine Angew. Math. 315 (1980), 40-52.

6. H. Davenport, Simultaneous Diophantine approximations, Proc. London Math. Soc. 2 (1952), 403-416.

7. Simultaneous Diophantine approximation, Proc. Internat. Congr. Math. (Amsterdam 1954), vol. III, Noordhoff, Groningen; North-Holland, Amsterdam, 1956, pp. 9-12.

8. H. Davenport and K. Mahler, Simultaneous Diophantine approximation, Duke Math. J. 13 (1946), $105-111$.

9. H. Davenport and W. Schmidt, Approximation to real numbers by quadratic irrationals, Acta Arith. 13 (1967), 169-176.

10. A theorem on linear forms, Acta Arith. 14 (1968), 209-223.

11. E. Dubois, F-best approximation of zero by a cubic linear form: Calculation of the fundamental unit of a not totally real cubic field, Proc. Queen's Number Theory Conf., 1979 (P. Ribenboim, ed.), Queen's Papers in Pure and Appl. Math., no. 54, Queen's University, Kingston, 1980, pp. 205-222.

12. E. Dubois and G. Rhin, Approximations simultanees de deux nombres reels, Sem. Delange-PisotPoitou, 20e annee 1978/1979, Theorie des Nombres, Fasc. 1, Exp. No. 9, Secretariat Math., Paris, 1980.

13. W. Jurkat, W. Kratz and A. Peyerimhoff, On best two dimensional Dirichlet approximations and their algorithmic calculation, Math. Ann. 244 (1979), 1-32.

14. J. C. Lagarias, Some new results in simultaneous Diophantine approximation, Proc. Queen's Number Theory Conf., 1979 (P. Ribenboim, ed), Queen's Papers in Pure and Appl. Math., no. 54, Queens University, Kingston, 1980, pp. 453-474.

15. __ Best simultaneous Diophantine approximations. II. Behavior of consecutive best approximations, Pacific J. Math. (to appear).

16. B Best simultaneous Diophantine approximations. III. Approximations to a basis of a non-totally real cubic field, in preparation.

17. S. Lang, Introduction to Diophantine approximation, Addison-Wesley, Reading, Mass., 1966.

Bell Laboratories, Murray Hill, New Jersey 07974 